\title{
Derivation of generalized Einstein's equations of gravitation in inertial systems based on a sink flow model of particles
}

\author{
Xiao-Song Wang \\ Institute of Mechanical and Power Engineering, Henan Polytechnic University, Jiaozuo, Henan Province, 454000, China
}

(Dated: Jul. 19, 2019)

\begin{abstract}
J. C. Maxwell, B. Riemann and H. Poincaré have proposed the idea that all microscopic particles are sink flows in a fluidic aether. Following this research program, a previous theory of gravitation based on a mechanical model of vacuum and a sink flow model of particles is generalized by methods of special relativistic continuum mechanics. In inertial coordinate systems, we construct a tensorial potential which satisfies the wave equations. Inspired by the equations of motion of a test particle, a definition of a metric tensor of a Riemannian spacetime is introduced. Applying Fock's theorem, generalized Einstein's equations in inertial systems are derived based on some assumptions. These equations reduce to Einstein's equations in case of weak field in harmonic coordinate systems.
\end{abstract}

Keywords: Einstein's equations; gravitation; general relativity; sink; gravitational aether.

\section{INTRODUCTION}

The Einstein's equations of gravitational fields in the theory of general relativity can be written as [1, 2]

$$
R_{\mu \nu}-\frac{1}{2} g_{\mu \nu} R=-\kappa T_{\mu \nu}^{m}
$$

where $g_{\mu \nu}$ is the metric tensor of a Riemannian spacetime, $R_{\mu \nu}$ is the Ricci tensor, $R \equiv g^{\mu \nu} R_{\mu \nu}$ is the scalar curvature, $g^{\mu \nu}$ is the contravariant metric tensor, $\kappa$ is a constant, $T_{\mu \nu}^{m}$ is the energy-momentum tensor of a matter system.

The Einstein's equations (11) are fundamental assumptions in the theory of general relativity [1, 2]. It is remarkable that Einstein's theory of general relativity, born in the year of 1915, has held up under every experimental test, refers to, for instance, [3].

There is a long history of researches of derivations or interpretations of Einstein's theory of general relativity. For instance, C. Misner et al. introduce six derivations of the Einstein's equations Eqs. (11) in their great book ([2], p. 417). S. Weinberg proposed two derivations ([1], p. 151).

However, these theories still face the following difficulties. (1) Attempts to reconcile the theory of general relativity and quantum mechanics have met some mathematical difficulties (4],p. 101); (2) The cosmological constant problem is still a puzzle, refers to, for instance, [5]; (3) The existence of black hole is still controversy, refers to, for instance, [6]; (4) Theoretical interpretation of P. A. M. Dirac's dimensionless large number ([7], p. 73) is still open; (5) The existences and characters of dark matter and dark energy are still controversy, refers to, for instance, [8]; (6) The existence and characters of gravitational aether are still not clear, refers to, for instance, [9]; (7) Whether Newton's gravitational constant $\gamma_{N}$ depends on time and space is still not clear [10]; (8) Whether the speed of light in vacuum depends on time or space is controversy, refers to, for instance, [11].

Furthermore, there exists some other problems related to the theories of gravity, for instance, the definition of inertial system, origin of inertial force, the velocity of the propagation of gravity [12], the velocity of individual photons [13, 14], unified field theory, etc.

The purpose of this manuscript is to propose a derivation of the Einstein's equations (1) in inertial coordinate systems based on a mechanical model of vacuum and a sink flow model of particles [15].

\section{INTRODUCTION OF A PREVIOUS THEORY OF GRAVITATION BASED ON A SINK FLOW MODEL OF PARTICLES BY METHODS OF CLASSICAL FLUID MECHANICS}

The idea that all microscopic particles are sink flows in a fluidic substratum is not new. For instance, in order to compare fluid motions with electric fields, J. C. Maxwell introduced an analogy between source or sink flows and electric charges ([16], p. 243). B. Riemann speculates that:" I make the hypothesis that space is filled with a substance which continually flows into ponderable atoms, and vanishes there from the world of phenomena, the corporeal world" ([17], p. 507). H. Poincaré also suggests that matters may be holes in fluidic aether ([18], p. 171). A. Einstein and L. Infeld said ([19], p. 256-257):" Matter is where the concentration of energy is great, field where the concentration of energy is small. ... What impresses our senses as matter is really a great concentration of energy into a comparatively small space. We could regard matter as the regions in space where the field is extremely strong."

Following these researchers, we suppose that all the microscopic particles were made up of a kind of elementary sinks of a fluidic medium filling the space [15]. Thus, Newton's law of gravitation is derived by methods of hydrodynamics based on the fluid model of vacuum and the sink flow model of particles [15].

We briefly introduce this theory of gravitation [15]. Suppose that there exists a fluidic medium filling the interplanetary vacuum. For convenience, we may call this medium as the $\Omega(0)$ substratum, or gravitational aether, 


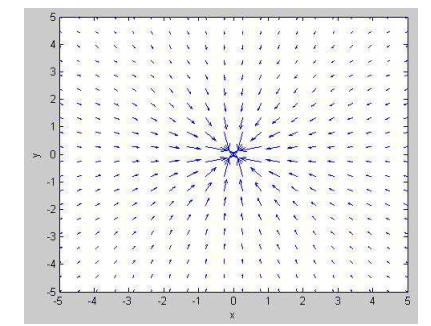

FIG. 1: an illustration of the velocity field of a sink flow.

or tao 15]. Suppose that the following conditions are valid: (1) the $\Omega(0)$ substratum is an ideal fluid; (2) the ideal fluid is irrotational and barotropic; (3) the density of the $\Omega(0)$ substratum is homogeneous; (4) there are no external body forces exerted on the fluid; (5) the fluid is unbounded and the velocity of the fluid at the infinity is approaching to zero.

An illustration of the velocity field of a sink flow can be found in Figure 1. If a point source is moving with a velocity $\mathbf{v}_{s}$, then there is a force [15]

$$
\mathbf{F}_{Q}=-\rho_{0} Q\left(\mathbf{u}-\mathbf{v}_{s}\right)
$$

is exerted on the source by the fluid, where $\rho_{0}$ is the density of the fluid, $Q$ is the strength of the source, $\mathbf{u}$ is the velocity of the fluid at the location of the source induced by all means other than the source itself.

We suppose that all the elementary sinks were created simultaneously [15]. For convenience, we may call these elementary sinks as monads. The initial masses and the strengths of the monads are the same. Suppose that (1) $\mathbf{v}_{i} \ll \mathbf{u}_{i}, i=1,2$, where $\mathbf{v}_{i}$ is the velocity of the particle with mass $m_{i}, \mathbf{u}_{i}$ is the velocity of the $\Omega(0)$ substratum at the location of the particle with mass $m_{i}$ induced by the other particle; (2) there are no other forces exerted on the particles, then the force $\mathbf{F}_{12}(t)$ exerted on the particle with mass $m_{2}(t)$ by the velocity field of $\Omega(0)$ substratum induced by the particle with mass $m_{1}(t)$ is [15]

$$
\mathbf{F}_{12}(t)=-\gamma_{N}(t) \frac{m_{1}(t) m_{2}(t)}{r^{2}} \hat{\mathbf{r}}_{12},
$$

where $\gamma_{N}$ is Newton's gravitational constant, $\hat{\mathbf{r}}_{12}$ denotes the unit vector directed outward along the line from the particle with mass $m_{1}(t)$ to the particle with mass $m_{2}(t)$, $r$ is the distance between the two particles, $m_{0}(t)$ is the mass of monad at time $t,-q_{0}\left(q_{0}>0\right)$ is the strength of a monad, and

$$
\gamma_{N}(t)=\frac{\rho_{0} q_{0}^{2}}{4 \pi m_{0}^{2}(t)}
$$

For continuously distributed matter, we have

$$
\frac{\partial \rho_{0}}{\partial t}+\nabla \cdot\left(\rho_{0} \mathbf{u}\right)=-\rho_{0} \rho_{s}
$$

where $\mathbf{u}$ is the velocity of the $\Omega(0)$ substratum, $\nabla=$ $\mathbf{i} \partial / \partial x+\mathbf{j} \partial / \partial y+\mathbf{k} \partial / \partial z$ is the nabla operator introduced by Hamilton, $\mathbf{i}, \mathbf{j}, \mathbf{k}$ are basis vectors, $-\rho_{s}\left(\rho_{s}>0\right)$ is the density of continuously distributed sinks, i.e.,

$$
-\rho_{s}=\lim _{\triangle V \rightarrow 0} \frac{\triangle Q}{\triangle V}
$$

where $\triangle Q$ is the source strength of the continuously distributed matter in the volume $\Delta V$ of the $\Omega(0)$ substratum.

Since the $\Omega(0)$ substratum is homogeneous, i.e., $\partial \rho_{0} / \partial t=\partial \rho_{0} / \partial x=\partial \rho_{0} / \partial y=\partial \rho_{0} / \partial z=0$, and irrotational, i.e., $\nabla \times \mathbf{u}=0$, Eq. (5) can be written as [20]

$$
\nabla^{2} \varphi=-\rho_{s}
$$

where $\varphi$ is a velocity potential such that $\mathbf{u}=\nabla \varphi, \nabla^{2}=$ $\partial^{2} / \partial x^{2}+\partial^{2} / \partial y^{2}+\partial^{2} / \partial z^{2}$ is the Laplace operator.

We introduce the following definitions

$$
\Phi=\frac{\rho_{0} q_{0}}{m_{0}} \varphi, \quad \rho_{m}=\frac{m_{0} \rho_{s}}{q_{0}},
$$

where $\rho_{m}$ denotes the mass density of continuously distributed particles.

Using Eq. (8) and Eq. (44), Eq. (7) can be written as

$$
\nabla^{2} \Phi=-4 \pi \gamma_{N} \rho_{m}
$$

\section{A MECHANICAL MODEL OF VACUUM}

According to our previous paper 21] we suppose that vacuum is filled with a kind of continuously distributed material which may be called $\Omega(1)$ substratum or electromagnetic aether. Maxwell's equations in vacuum are derived by methods of continuum mechanics based on this mechanical model of vacuum and a source and sink flow model of electric charges [21]. We speculate that the electromagnetic aether may also generate gravity. Thus, we introduce the following assumption.

Assumption 1 The particles that constitute the $\Omega(1)$ substratum, or the electromagnetic aether, are sinks in the $\Omega(0)$ substratum.

Then, according to the previous theory of gravitation [15], these $\Omega(1)$ particles gravitate with each other and also attract with matters. Thus, vacuum is composed of at least two kinds of interacting substratums, i.e., the gravitational aether $\Omega(0)$ and the electromagnetic aether $\Omega(1)$.

From Eq. (2), we see that there exists a following universal damping force $\mathbf{F}_{d}=-\rho_{0} q_{0} m \mathbf{v}_{p} / m_{0}$ exerted on each particle by the $\Omega(0)$ substratum [15], where $\mathbf{v}_{p}$ is the velocity of the particle. Based on this universal damping force $\mathbf{F}_{d}$ and some assumptions, we derive a generalized Schrödinger equation for microscopic particles 22]. For convenience, we may call these theories [15, 21, 22] as the theory of vacuum mechanics. 


\section{CONSTRUCTION OF A LAGRANGIAN FOR FREE FIELDS OF THE $\Omega(0)$ SUBSTRATUM BASED ON A TENSORIAL POTENTIAL IN THE GALILEAN COORDINATES}

There exists some approaches ([23], page vii; 2], p. 424), which regards Einstein's general relativity as a special relativistic field theory in an unobservable flat spacetime, to derive the Einstein's equations (11). However, these theories can not provide a physical definition of the tensorial potential of gravitational fields, refers to, for instance, [2, 24, 25]. Thus, similar to the theory of general relativity, these theories may be regarded as phenomenological theories of gravitation.

Inspired by these special relativistic field theories of gravitation, we explore the possibility of establishing a similar theory based on the theory of vacuum mechanics [15, 21, 22]. Thus, first of all, we need to construct a Lagrangian for free fields of the $\Omega(0)$ substratum based on a tensorial potential in the Galilean coordinates. In this section, we will regard the $\Omega(0)$ substratum in the previous theory of gravitation [15] as a special relativistic fluid. Then, we will study the $\Omega(0)$ substratum by methods of special relativistic continuum mechanics [26].

In this article, we adopt the mathematical framework of the theory of special relativity [1]. However, the physical interpretation of the mathematics of the theory of special relativity may be different from Einstein's theory. It is known that Maxwell's equations are valid in the frames of reference that attached to the $\Omega(1)$ substratum [21]. We introduce a Cartesian coordinate system $\{0, x, y, z\}$ for a three-dimensional Euclidean space that attached to the $\Omega(1)$ substratum. Let $\{0, t\}$ be a onedimensional time coordinate. We denote this reference frame as $S_{\Omega(1)}$.

Based on the Maxwell's equations, the law of propagation of an electromagnetic wave front in this reference frame $S_{\Omega(1)}$ can be derived and can be written as ([27],p. 13)

$$
\frac{1}{c^{2}}\left(\frac{\partial \omega}{\partial t}\right)^{2}-\left(\frac{\partial \omega}{\partial x}\right)^{2}-\left(\frac{\partial \omega}{\partial y}\right)^{2}-\left(\frac{\partial \omega}{\partial z}\right)^{2}=0
$$

where $\omega(t, x, y, z)$ is an electromagnetic wave front, $c$ is the velocity of light in the reference frame $S_{\Omega(1)}$.

An electromagnetic wave front is a characteristics. According to Fock's theorem of characteristics (27], p. 432), we obtain the following metric tensor $\eta_{\alpha \beta}=$ $\operatorname{diag}\left[c^{2},-1,-1,-1\right]$ of a Minkowski spacetime for vacuum (28], p. 57), where $c$ is the speed of light in vacuum.

For convenience, we introduce the following Galilean coordinate system

$$
x^{0} \equiv c t, \quad x^{1} \equiv x, \quad x^{2} \equiv y, \quad x^{3} \equiv z .
$$

We will use Greek indices $\alpha, \beta, \mu, \nu$, etc., denote the range $\{0,1,2,3\}$ and use Latin indices $i, j, k$, etc., denote the range $\{1,2,3\}$. We will use Einstein's summation convention, that is, any repeated Greek superscript or subscript appearing in a term of an equation is to be summed from 0 to 3 . We introduce the following definition of spacetime interval

$$
d s^{2}=\eta_{\mu \nu} d x^{\mu} d x^{\nu}
$$

where $\eta_{\mu \nu}$ is the metric tensor of the Minkowski spacetime defined by $\eta_{\mu \nu}=\operatorname{diag}[1,-1,-1,-1]$.

Suppose that the $\Omega(0)$ substratum is an incompressible viscous fluid. Then, there is no elastic deformations in the fluid and the internal stress states depend on the instantaneous velocity field. Thus, we can choose the reference frame $S_{\Omega(1)}$ as the co-moving coordinate system. The internal energy $U$ is the sum of the internal elastic energy $U_{e}$ and the dissipative energy $U_{d}$, i.e., $U=U_{e}+U_{d}$. Since there is no elastic deformations in the fluid, we have $U_{e}=0$. We introduce the following definition of deviatoric tensor of strain rate $\dot{\gamma}_{j}^{i}([29]$,p. 331)

$$
\dot{\gamma}_{j}^{i}=\dot{S}_{j}^{i}-\dot{S}_{k}^{k} \delta_{j}^{i}
$$

where $\dot{S}_{j}^{i}$ is the tensor of strain rate, $\dot{S}_{k}^{k}$ is the rate of volume change, $\delta_{j}^{i}$ is the Kronecker delta.

Suppose that the rate of dissipative energy $\dot{U}_{d}$ is the Rayleigh type, then, we have ([29],p. 332)

$$
\dot{U}_{d}=\mu_{0} \dot{\gamma}_{j}^{i} \dot{\gamma}_{i}^{j}
$$

where $\mu_{0}$ is the coefficient of viscosity.

Since the $\Omega(0)$ substratum is incompressible, we have $\dot{S}_{k}^{k}=0$. Thus, from Eqs. (14) and Eqs. (13), we have

$$
\dot{U}_{d}=\mu_{0} \dot{S}_{j}^{i} \dot{S}_{i}^{j}
$$

In the low velocity limit, i.e., $u / c \ll 1$, where $u=|\mathbf{u}|$, the Lagrangian $L_{\Omega(0)}$ for free fields of the $\Omega(0)$ substratum can be written as $([29]$,p. 332)

$$
L_{\Omega(0)}=\frac{1}{2} \rho_{0} u^{2}+\int_{t_{0}}^{t} \dot{U}_{d}\left(\dot{S}_{j}^{i}\right) d t,
$$

where $u=|\mathbf{u}|, t_{0}$ is an initial time.

Suppose that the $\Omega(0)$ substratum is a Newtonian fluid and the stress tensor $\sigma_{j}^{i}$ is symmetric, then we have ([30],p. 46)

$$
\sigma_{j}^{i}=-p \delta_{j}^{i}+2 \mu_{0} \dot{S}_{j}^{i}
$$

where $p$ is the pressure of the $\Omega(0)$ substratum.

Using Eqs. (17) and Eqs. (15), Eqs. (16) can be written as

$$
L_{\Omega(0)}=\frac{1}{2} \rho_{0} u^{2}+\int_{t_{0}}^{t}\left(\sigma_{j}^{i}+p \delta_{j}^{i}\right) \frac{\dot{S}_{i}^{j}}{2} d t
$$

For a macroscopic observer, the relaxation time $t_{\varepsilon}$ of the $\Omega(0)$ substratum is so small that the tensor of strain rate $\dot{S}_{j}^{i}$ may be regarded as a slow varying function of 
time, i.e., $\partial \dot{S}_{j}^{i} / \partial t \ll 1$. Thus, in a small time interval $\left[t_{0}, t\right]$, we have $\dot{S}_{j}^{i} \geq 0$, or, $\dot{S}_{j}^{i} \leq 0$. Then, it is possible to choose a value $\bar{\sigma}_{j}^{i}+\bar{p} \delta_{j}^{i}$ of $\sigma_{j}^{i}+p \delta_{j}^{i}$ in the time interval $\left[t_{0}, t\right]$ such that Eqs. (18) can be written as

$$
L_{\Omega(0)}=\frac{1}{2} \rho_{0} u^{2}+\left(\bar{\sigma}_{j}^{i}+\bar{p} \delta_{j}^{i}\right) \int_{t_{0}}^{t} \frac{\dot{S}_{i}^{j}}{2} d t .
$$

We introduce the following definition

$$
\psi_{i j} \triangleq \int_{t_{0}}^{t} \frac{\dot{S}_{i j}}{2 f_{0}} d t
$$

where $f_{0}$ is a parameter to be determined.

Using Eqs. (20), Eqs. (19) can be written as

$$
L_{\Omega(0)}=\frac{1}{2} \rho_{0} u^{2}+f_{0} \psi_{i}^{j}\left(\bar{\sigma}_{j}^{i}+\bar{p} \delta_{j}^{i}\right) .
$$

Since the coefficient of viscosity $\mu_{0}$ of the $\Omega(0)$ substratum may be very small, we introduce the following assumption.

Assumption 2 In the low velocity limit, i.e., $u / c \ll 1$, where $u=|\boldsymbol{u}|, \boldsymbol{u}$ is the velocity of the $\Omega(0)$ substratum, we suppose that $\mu_{0} \approx 0$ and we have the following conditions

$$
\psi_{i j} \approx 0, \quad \partial_{\mu} \psi_{i j} \approx 0, \quad \partial_{\mu} \partial_{\nu} \psi_{i j} \approx 0,
$$

where

$$
\partial_{\mu} \equiv\left(\frac{\partial}{\partial x^{0}}, \frac{\partial}{\partial x^{1}}, \frac{\partial}{\partial x^{2}}, \frac{\partial}{\partial x^{3}}\right) .
$$

According to the Stokes-Helmholtz resolution theorem, refers to, for instance, 31], every sufficiently smooth vector field can be decomposed into irrotational and solenoidal parts. Thus, there exists a scalar function $\varphi$ and a vector function $\mathbf{R}$ such that the velocity field $\mathbf{u}$ of the $\Omega(0)$ substratum can be represented by [31]

$$
\mathbf{u}=\nabla \varphi+\nabla \times \mathbf{R},
$$

where $\nabla \times \varphi=0, \nabla \cdot \mathbf{R}=0$.

We introduce the following definition of a vector function $\vec{\xi}$

$$
\frac{\partial \vec{\xi}}{\partial(c t)}=\nabla \times \mathbf{R}
$$

Putting Eq. (25) into Eq. (24), we have

$$
\mathbf{u}=\nabla \varphi+\frac{\partial \vec{\xi}}{\partial(c t)}
$$

Based on Assumption 2 and using Eq. (8) and Eq. (26), Eq. (21) can be written as

$$
\begin{aligned}
L_{\Omega(0)} & =\frac{1}{2} \rho_{0} u^{2}=\frac{1}{2} \rho_{0}\left(\nabla \varphi+\frac{\partial \vec{\xi}}{\partial(c t)}\right)^{2} \\
& =\frac{1}{2} \rho_{0}\left(\frac{m_{0}}{\rho_{0} q_{0}} \nabla \Phi+\frac{\partial \vec{\xi}}{\partial(c t)}\right)^{2} .
\end{aligned}
$$

We introduce the following definitions

$$
\begin{aligned}
& \psi_{00}=-a_{00} \Phi, \quad \psi_{0 i}=\psi_{i 0}=a_{0 i} \xi_{i}, \\
& \vec{\psi}_{0}=\psi_{01} \mathbf{i}+\psi_{02} \mathbf{j}+\psi_{03} \mathbf{k} .
\end{aligned}
$$

where $a_{00}>0$ and $a_{0 i}>0$ are 4 parameters to be determined.

Eqs. (28) and Eqs. (20) have defined a rank 2 symmetric tensor $\psi_{\mu \nu}$. We require that for some special values of $a_{00}$ and $a_{0 i}$, Eq. (27) can be written as

$$
\begin{aligned}
L_{\Omega(0)} & =\left(\frac{m_{0}}{q_{0} \sqrt{2 \rho_{0}}} \frac{1}{a_{00}} \nabla \psi_{00}-\sqrt{\frac{\rho_{0}}{2}} \frac{\partial\left(\psi_{0 i} / a_{0 i}\right)}{\partial(c t)} \mathbf{e}^{i}\right)^{2} \\
& \equiv\left(\nabla \psi_{00}-\frac{\partial \vec{\psi}_{0}}{\partial(c t)}\right)^{2},
\end{aligned}
$$

where $\mathbf{e}^{1} \equiv \mathbf{i}, \mathbf{e}^{2} \equiv \mathbf{j}, \mathbf{e}^{3} \equiv \mathbf{k}$.

Comparing the left- and right-hand parts of Eq. (30), we have

$$
a_{00}=\sqrt{\frac{m_{0}^{2}}{2 \rho_{0} q_{0}^{2}}}, \quad a_{0 i}=\sqrt{\frac{\rho_{0}}{2}} .
$$

In order to construct the Lagrangian $L_{\Omega(0)}$ described in Eq. (30) based on the tensorial potential $\psi_{\mu \nu}$, we should consider all the possible products of derivatives of the tensor $\psi_{\mu \nu}$. If we require that the two tensor indices of $\psi_{\mu \nu}$ are different from each other and the two tensor indices of $\psi_{\mu \nu}$ are different from the derivative index, we have the following two possible products ([23], p. 43):

$$
L_{1}=\partial_{\sigma} \psi_{\mu \nu} \partial^{\sigma} \psi^{\mu \nu}, \quad L_{2}=\partial_{\sigma} \psi_{\mu \nu} \partial^{\mu} \psi^{\sigma \nu},
$$

where $\psi^{\mu \nu}=\eta^{\mu \lambda} \eta^{\nu \sigma} \psi_{\lambda \sigma}$ is the corresponding contravariant tensor of $\psi_{\mu \nu}$.

If there are two indices of $\psi_{\mu \nu}$ which are equal, or one of the indices of $\psi_{\mu \nu}$ is the same as the derivative index, we may have the following three possible products ([23], p. 43):

$$
\begin{aligned}
& L_{3}=\partial_{\nu} \psi^{\mu \nu} \partial_{\sigma} \psi_{\mu}^{\sigma}, \quad L_{4}=\partial^{\mu} \psi_{\mu \nu} \partial^{\nu} \psi, \\
& L_{5}=\partial_{\lambda} \psi \partial^{\lambda} \psi .
\end{aligned}
$$

where $\psi$ is the trace of $\psi_{\mu \nu}$, i.e., $\psi \equiv \psi_{\lambda}^{\lambda}=\eta_{\alpha \beta} \psi^{\alpha \beta}$,

$$
\partial^{\mu} \equiv \eta^{\mu \nu} \partial_{\nu}=\left(\frac{\partial}{\partial x^{0}},-\frac{\partial}{\partial x^{1}},-\frac{\partial}{\partial x^{2}},-\frac{\partial}{\partial x^{3}}\right) .
$$

$L_{3}$ may be omitted because it can be converted to $L_{2}$ by integration by parts (23], p. 43).

Proposition 1 Suppose that we have the following conditions

$$
\frac{\partial \psi_{00}}{\partial(c t)} \approx 0, \quad \frac{\partial \psi_{0 i}}{\partial x^{j}} \approx 0
$$

If we set

$$
c_{1}=\frac{1}{2}, \quad c_{2}=-2, \quad c_{4}=-6, \quad c_{5}=-\frac{3}{2},
$$


then we have

$c_{1} L_{1}+c_{2} L_{2}+c_{4} L_{4}+c_{5} L_{5} \approx\left(\nabla \psi_{00}-\frac{\partial \vec{\psi}_{0}}{\partial(c t)}\right)^{2}=\frac{1}{2} \rho_{0} u^{2}$.

Proof of Proposition 1. Based on Eqs. (22) and Eqs. (36) and noticing $\psi^{00}=\psi_{00}, \psi^{0 i}=-\psi_{0 i}$, we have

$$
\begin{gathered}
L_{1} \approx-\left(\nabla \psi_{00}\right)^{2}-2\left(\frac{\partial \vec{\psi}_{0}}{\partial(c t)}\right)^{2}, \\
L_{2} \approx-2\left(\nabla \psi_{00}\right) \cdot \frac{\partial \vec{\psi}_{0}}{\partial(c t)}-\left(\frac{\partial \vec{\psi}_{0}}{\partial(c t)}\right)^{2}, \\
L_{3} \approx\left(\nabla \psi_{00}\right) \cdot \frac{\partial \vec{\psi}_{0}}{\partial(c t)} \\
L_{4} \approx-\left(\nabla \psi_{00}\right)^{2} .
\end{gathered}
$$

Using Eqs. (39, 42) and Eqs. (37), we obtain Eq. (38).

Inspired by W. Thirring [24] and R. P. Feynman ([23], p. 43), we introduce the following assumption.

Assumption 3 The Lagrangian $L_{\Omega(0)}$ for free fields of the $\Omega(0)$ substratum can be written as

$$
L_{\Omega(0)}=c_{1} L_{1}+c_{2} L_{2}+c_{4} L_{4}+c_{5} L_{5}+L_{\text {more }},
$$

where $c_{1}=1 / 2, \quad c_{2}=-2, \quad c_{4}=-6, \quad c_{5}=-3 / 2$, $L_{\text {more }}$ denotes those terms involving more than two derivatives of $\psi_{\mu \nu}$.

\section{INTERACTION TERMS OF THE LAGRANGIAN OF A SYSTEM OF THE $\Omega(0)$ SUBSTRATUM, THE $\Omega(1)$ SUBSTRATUM AND MATTER}

In order to derive the field equations, we should explore the possible interaction terms of the Lagrangian of a system of the $\Omega(0)$ substratum, the $\Omega(1)$ substratum and matter. According to Assumption 2, the coefficient of viscosity $\mu_{0}$ of the $\Omega(0)$ substratum may be very small. Thus, we may regard the $\Omega(0)$ substratum as an ideal fluid approximately. Then from Eq. (24) we have $\mathbf{u}=\nabla \varphi$. Ignoring the damping force $\rho_{0} Q \mathbf{v}_{s}$ in Eq. (2) and using $\mathbf{u}=\nabla \varphi$, Eq. (2) can be written as

$$
\mathbf{F}_{Q}=-\rho_{0} Q \nabla \varphi
$$

A particle is modelled as a point sink of the $\Omega(0)$ substratum [15, 21, 22]. Thus, the interaction term of the Lagrangian of a system of the $\Omega(0)$ substratum and a particle can be written as

$$
L_{\text {int }}=\rho_{0} Q \varphi \text {. }
$$

Thus, the interaction term of the Lagrangian of a system of the $\Omega(0)$ substratum and continuously distributed particles can be written as

$$
L_{\text {int }}=-\rho_{0} \rho_{s} \varphi
$$

Putting Eq. (8) into Eq. (46), we have

$$
L_{\mathrm{int}}=-\rho_{m} \Phi
$$

The 00 term of the energy-momentum tensor $T_{\mu \nu}^{m}$ of a matter system is $T_{m}^{00}=\rho_{m} c^{2}$. Thus, using Eqs. (28), Eq. (47) can be written as

$$
L_{\text {int }}=f_{0} \psi_{00} T_{m}^{00},
$$

where

$$
f_{0}=\frac{1}{a_{00} c^{2}}
$$

From Eq. (49), Eq. (31) and Eq. (44), we have

$$
f_{0}=\sqrt{\frac{2 \rho_{0} q_{0}^{2}}{m_{0}^{2} c^{4}}}=\sqrt{\frac{8 \pi \gamma_{N}}{c^{4}}}, \quad \frac{1}{a_{00}^{2}}=8 \pi \gamma_{N} .
$$

Inspired by Eq. (48) and Eq. (21), we introduce the following assumption.

Assumption 4 The interaction terms of the Lagrangian of a system of the $\Omega(0)$ substratum, the $\Omega(1)$ substratum and matter can be written in the following form:

$$
L_{\mathrm{int}}=f_{0} \psi_{\mu \nu} T_{m}^{\mu \nu}+f_{0} \psi_{\mu \nu} T_{\Omega(1)}^{\mu \nu}+O\left[\left(f_{0} \psi_{\mu \nu}\right)^{2}\right],
$$

where $T_{m}^{\mu \nu}$ and $T_{\Omega(1)}^{\mu \nu}$ are the contravariant energymomentum tensors of the system of the matter and the $\Omega(1)$ substratum respectively, $O\left[\left(f_{0} \psi_{\mu \nu}\right)^{2}\right]$ denotes those terms which are small quantities of the order of $\left(f_{0} \psi_{\mu \nu}\right)^{2}$.

\section{DERIVATION OF THE FIELD EQUATIONS}

Based on Assumptions 3 and 4, the total Lagrangian $L_{\text {tot }}$ of a system of the $\Omega(0)$ substratum, the $\Omega(1)$ substratum and matter can be written as

$$
\begin{aligned}
L_{\text {tot }}= & \frac{1}{2} \partial_{\lambda} \psi_{\mu \nu} \partial^{\lambda} \psi^{\mu \nu}-2 \partial_{\lambda} \psi_{\mu \nu} \partial^{\mu} \psi^{\lambda \nu}-6 \partial^{\mu} \psi_{\mu \nu} \partial^{\nu} \psi \\
& -\frac{3}{2} \partial_{\lambda} \psi \partial^{\lambda} \psi+L_{\text {more }}+f_{0} \psi_{\mu \nu}\left(T_{m}^{\mu \nu}+T_{\Omega(1)}^{\mu \nu}\right) \\
& +O\left[\left(f_{0} \psi_{\mu \nu}\right)^{2}\right] .
\end{aligned}
$$

Theorem 1 If we ignore those terms which are small quantities of the order of $\left(f_{0} \psi_{\mu \nu}\right)^{2}$ and those terms involving more than two derivatives of $\psi_{\mu \nu}$ in Eq. (52), i.e., $O\left[\left(f_{0} \psi_{\mu \nu}\right)^{2}\right]$ and $L_{\text {more }}$, then the field equations for the total Lagrangian $L_{\text {tot }}$ in Eq. (52) can be written as

$$
\begin{aligned}
& \partial_{\sigma} \partial^{\sigma} \psi_{\alpha \beta}-2\left(\partial^{\sigma} \partial_{\alpha} \psi_{\beta \sigma}+\partial^{\sigma} \partial_{\beta} \psi_{\alpha \sigma}\right)-6\left(\eta_{\alpha \beta} \partial_{\sigma} \partial_{\lambda} \psi^{\sigma \lambda}\right. \\
& \left.+\partial_{\alpha} \partial_{\beta} \psi\right)-3 \eta_{\alpha \beta} \partial_{\sigma} \partial^{\sigma} \psi=f_{0}\left(T_{\alpha \beta}^{m}+T_{\alpha \beta}^{\Omega(1)}\right) .
\end{aligned}
$$


Proof of Theorem 1. Starting from the Lagrangian in Eq. (52), we have the following Euler-Lagrange equations 32 ]

$$
\frac{\partial L_{\mathrm{tot}}}{\partial \psi^{\alpha \beta}}-\frac{\partial}{\partial x^{\sigma}}\left(\frac{\partial L_{\mathrm{tot}}}{\partial\left(\partial_{\sigma} \psi^{\alpha \beta}\right)}\right)=0 .
$$

We can verify the following results $(23]$, p. 43; 24] $)$

$$
\begin{aligned}
\frac{\partial}{\partial x^{\sigma}}\left[\frac{\partial\left(\partial_{\lambda} \psi_{\mu \nu} \partial^{\lambda} \psi^{\mu \nu}\right)}{\partial\left(\partial_{\sigma} \psi^{\alpha \beta}\right)}\right] & =2 \partial_{\sigma} \partial^{\sigma} \psi_{\alpha \beta} \\
\frac{\partial}{\partial x^{\sigma}}\left[\frac{\partial\left(\partial_{\lambda} \psi_{\mu \nu} \partial^{\mu} \psi^{\lambda \nu}\right)}{\partial\left(\partial_{\sigma} \psi^{\alpha \beta}\right)}\right] & =\partial^{\sigma} \partial_{\alpha} \psi_{\beta \sigma}+\partial^{\sigma} \partial_{\beta} \psi_{\alpha \sigma}, \\
\frac{\partial}{\partial x^{\sigma}}\left[\frac{\partial\left(\partial^{\mu} \psi_{\mu \nu} \partial^{\nu} \psi\right)}{\partial\left(\partial_{\sigma} \psi^{\alpha \beta}\right)}\right] & =\partial_{\alpha} \partial_{\beta} \psi+\eta_{\alpha \beta} \partial_{\sigma} \partial_{\lambda} \psi^{\sigma \lambda} \\
\frac{\partial}{\partial x^{\sigma}}\left[\frac{\partial\left(\partial_{\lambda} \psi \partial^{\lambda} \psi\right)}{\partial\left(\partial_{\sigma} \psi^{\alpha \beta}\right)}\right] & =2 \eta_{\alpha \beta} \partial_{\sigma} \partial^{\sigma} \psi \\
\frac{\partial L_{\text {tot }}}{\partial \psi^{\alpha \beta}} & =f_{0}\left(T_{\alpha \beta}^{m}+T_{\alpha \beta}^{\Omega(1)}\right)
\end{aligned}
$$

Putting Eq. (52) into Eqs. (154) and using Eqs. (55),59), we obtain Eqs. (53).

For convenience, we introduce the following notation

$$
\begin{aligned}
\Psi^{\mu \nu}= & \partial_{\lambda} \partial^{\lambda} \psi^{\mu \nu}-2 \partial_{\lambda} \partial^{\mu} \psi^{\nu \lambda}-2 \partial_{\lambda} \partial^{\nu} \psi^{\mu \lambda} \\
& -6 \eta^{\mu \nu} \partial_{\sigma} \partial_{\lambda} \psi^{\sigma \lambda}-6 \partial^{\mu} \partial^{\nu} \psi-3 \eta^{\mu \nu} \partial_{\lambda} \partial^{\lambda} \psi \cdot(60)
\end{aligned}
$$

Thus, the field equations Eqs. (53) can be written as

$$
\Psi^{\mu \nu}=f_{0}\left(T_{m}^{\mu \nu}+T_{\Omega(1)}^{\mu \nu}\right) .
$$

We introduce the following definition of the total energy-momentum tensor $T^{\mu \nu}$ of the system of the matter, the $\Omega(1)$ substratum and the $\Omega(0)$ substratum

$$
T^{\mu \nu}=T_{m}^{\mu \nu}+T_{\Omega(1)}^{\mu \nu}+T_{\Omega(0)}^{\mu \nu},
$$

where $T_{\Omega(0)}^{\mu \nu}$ is the energy-momentum tensor of the $\Omega(0)$ substratum.

Adding the term $f_{0} T_{\Omega(0)}^{\mu \nu}$ on both sides of Eqs. (61) and using Eqs. (62), the field equations Eqs. (61) can be written as

$$
\Psi^{\mu \nu}+f_{0} T_{\Omega(0)}^{\mu \nu}=f_{0} T^{\mu \nu} .
$$

For the total system of matter, the $\Omega(1)$ substratum and the $\Omega(0)$ substratum, the law of conservation of energy and momentum is ([26], p. 163; 28], p. 155)

$$
\partial_{\mu} T^{\mu \nu}=0 .
$$

Comparing Eqs. (64) and Eqs. (63), we have

$$
\partial_{\mu}\left(\Psi^{\mu \nu}+f_{0} T_{\Omega(0)}^{\mu \nu}\right)=0 .
$$

Noticing Eqs. (555(59), we introduce the following notation $([23]$, p. 43$)$

$$
\begin{aligned}
H^{\mu \nu}= & f_{1} \partial_{\lambda} \partial^{\lambda} \psi^{\mu \nu}+f_{2}\left(\partial_{\lambda} \partial^{\mu} \psi^{\nu \lambda}+\partial_{\lambda} \partial^{\nu} \psi^{\mu \lambda}\right) \\
& +f_{3}\left(\partial^{\mu} \partial^{\nu} \psi+\eta^{\mu \nu} \partial_{\sigma} \partial_{\lambda} \psi^{\sigma \lambda}\right) \\
& +f_{4} \eta^{\mu \nu} \partial_{\lambda} \partial^{\lambda} \psi
\end{aligned}
$$

where $f_{i}, i=1,2,3,4$ are 4 arbitrary parameters.

If we require that

$$
\partial_{\mu} H^{\mu \nu}=0,
$$

then, we can verify the following relationships ([23], p. $44 ; 24]$ )

$$
f_{1}+f_{2}=0, \quad f_{2}+f_{3}=0, \quad f_{3}+f_{4}=0 .
$$

We choose $f_{1}=1, f_{2}=-1, f_{3}=1, f_{4}=-1$ in Eqs. (66) and introduce the following notation

$$
\begin{aligned}
\Theta^{\mu \nu}= & \partial_{\lambda} \partial^{\lambda} \psi^{\mu \nu}-\left(\partial_{\lambda} \partial^{\mu} \psi^{\nu \lambda}+\partial_{\lambda} \partial^{\nu} \psi^{\mu \lambda}\right) \\
& +\left(\partial^{\mu} \partial^{\nu} \psi+\eta^{\mu \nu} \partial_{\sigma} \partial_{\lambda} \psi^{\sigma \lambda}\right)-\eta^{\mu \nu} \partial_{\lambda} \partial^{\lambda} \psi
\end{aligned}
$$

We can verify the following result ([23], p. 44; 24] $)$

$$
\partial_{\mu} \Theta^{\mu \nu}=0 .
$$

From Eqs. (70) and Eqs. (65), we have

$$
\partial_{\mu}\left(\frac{1}{f_{0}} \Psi^{\mu \nu}-\frac{b_{0}}{f_{0}} \Theta^{\mu \nu}+T_{\Omega(0)}^{\mu \nu}\right)=0 .
$$

where $b_{0}$ is an arbitrary parameter.

Noticing Eqs. (71), it is convenient for us to introduce the following definition of a tensor $T_{\omega}^{\mu \nu}$

$$
T_{\omega}^{\mu \nu}=\frac{1}{f_{0}} \Psi^{\mu \nu}-\frac{b_{0}}{f_{0}} \Theta^{\mu \nu}+T_{\Omega(0)}^{\mu \nu},
$$

where $b_{0}$ is a parameter to be determined.

From Eqs. (71), we have $\partial_{\mu} T_{\omega}^{\mu \nu}=0$. In the present stage, we have no idea about the physical meaning of the tensor $T_{\omega}^{\mu \nu}$. Later, once we have determined the value of the parameter $b_{0}$, we may explore the meaning of $T_{\omega}^{\mu \nu}$. Using Eqs. (72), the field equations Eqs. (63) can be written as

$$
\Theta^{\mu \nu}=\frac{f_{0}}{b_{0}}\left(T^{\mu \nu}-T_{\omega}^{\mu \nu}\right) .
$$

Now our task is to determine the parameter $b_{0}$ in the field equations (73). A natural idea is that the 00 component of Eqs. (73) reduces to the field equations Eqs. (9) in the case that the velocity of the $\Omega(0)$ substratum is much smaller than $c$, i.e., in the low velocity limit. Thus, it is necessary for us to introduce an estimation of the value of $T^{\mu \nu}-T_{\omega}^{\mu \nu}$ on the right hand side of Eqs. (73) in the low velocity limit. To this end, we introduce the following speculation about the interaction between the $\Omega(0)$ substratum and the $\Omega(1)$ substratum.

Assumption 5 In the low velocity limit, i.e., $u / c \ll 1$, where $u=|\boldsymbol{u}|, \boldsymbol{u}$ is the velocity of the $\Omega(0)$ substratum, the following relationship is valid

$$
T_{\Omega(1)}^{\mu \nu} \approx \frac{1}{f_{0}} \Psi^{\mu \nu}-\frac{b_{0}}{f_{0}} \Theta^{\mu \nu},
$$

where $b_{0}$ is a parameter to be determined. 
Therefore, using Eqs. (61) and Eqs. (74), we have the following estimation of $T_{m}^{\mu \nu}$ in the low velocity limit

$$
T_{m}^{\mu \nu} \approx \frac{b_{0}}{f_{0}} \Theta^{\mu \nu}
$$

Using Eqs. (63), Eqs. (72) and Eqs. (75), we have the following estimation of $T^{\mu \nu}-T_{\omega}^{\mu \nu}$ in the low velocity limit

$$
T^{\mu \nu}-T_{\omega}^{\mu \nu}=\frac{b_{0}}{f_{0}} \Theta^{\mu \nu} \approx T_{m}^{\mu \nu} .
$$

Theorem 2 Suppose that Assumption 5 is valid. Then, $b_{0}=-1$ and the field equations (53) can be written as

$$
\begin{aligned}
& \partial_{\lambda} \partial^{\lambda} \psi^{\mu \nu}-\partial_{\lambda} \partial^{\mu} \psi^{\nu \lambda}-\partial_{\lambda} \partial^{\nu} \psi^{\mu \lambda}+\partial^{\mu} \partial^{\nu} \psi \\
& +\eta^{\mu \nu} \partial_{\sigma} \partial_{\lambda} \psi^{\sigma \lambda}-\eta^{\mu \nu} \partial_{\lambda} \partial^{\lambda} \psi=-f_{0}\left(T^{\mu \nu}-T_{\omega}^{\mu \nu}\right)(77
\end{aligned}
$$

Proof of Theorem 2, Noticing Eqs. (69), the 00 component of the field equations (73) is

$$
\begin{aligned}
\partial_{\lambda} \partial^{\lambda} \psi^{00} & -2 \partial_{\lambda} \partial^{0} \psi^{0 \lambda}+\partial^{0} \partial^{0} \psi \\
& +\partial_{\sigma} \partial_{\lambda} \psi^{\sigma \lambda}-\partial_{\lambda} \partial^{\lambda} \psi=\frac{f_{0}}{b_{0}}\left(T^{00}-T_{\omega}^{00}\right)
\end{aligned}
$$

Take the trace of the field equations Eqs. (73), we have

$$
\partial_{\sigma} \partial_{\lambda} \psi^{\sigma \lambda}-\partial_{\lambda} \partial^{\lambda} \psi=\frac{f_{0}}{2 b_{0}}\left(T-T_{\omega}\right)
$$

where $T$ and $T_{\omega}$ are the traces of $T^{\mu \nu}$ and $T_{\omega}^{\mu \nu}$ respectively, i.e., $T \equiv T_{\lambda}^{\lambda}=\eta_{\alpha \beta} T^{\alpha \beta}, T_{\omega} \equiv T_{\omega \lambda}^{\lambda}=\eta_{\alpha \beta} T_{\omega}^{\alpha \beta}$.

Subtracting Eq. (79) from Eq. (78), we have

$$
\begin{aligned}
\partial_{\lambda} \partial^{\lambda} \psi^{00} & -2 \partial_{\lambda} \partial^{0} \psi^{0 \lambda}+\partial^{0} \partial^{0} \psi \\
& =\frac{f_{0}}{b_{0}}\left(T^{00}-\frac{T}{2}-T_{\omega}^{00}+\frac{T_{\omega}}{2}\right) .
\end{aligned}
$$

If the field is time-independent, then Eq. (80) reduces to

$$
-\nabla^{2} \psi^{00}=\frac{f_{0}}{b_{0}}\left(T^{00}-\frac{T}{2}-T_{\omega}^{00}+\frac{T_{\omega}}{2}\right) .
$$

According to Eqs. (76), we have the following estimations in the low velocity limit

$$
T^{00}-T_{\omega}^{00} \approx T_{m}^{00}=\rho_{m} c^{2}, \quad T-T_{\omega} \approx T_{m} \approx \rho_{m} c^{2},
$$

where $T_{m}$ is the trace of $T_{m}^{\mu \nu}$, i.e., $T_{m} \equiv \eta_{\alpha \beta} T_{m}^{\alpha \beta}$.

Using Eqs. (28), Eq. (31), Eq. (50) and Eqs. (82), Eq. (81) can be written as

$$
\nabla^{2} \Phi=\frac{1}{b_{0}} 4 \pi \gamma_{N} \rho_{m}
$$

Comparing Eq. (83) and Eq. (9), we obtain $b_{0}=-1$. Therefore, using Eqs. (69) and $b_{0}=-1$, the field equations Eqs. (73) can be written as Eqs. (777).
Now we discuss the physical meaning of $T_{\omega}^{\mu \nu}$. Noticing Eqs. (74) and Eqs. (72), we have the following estimation in the low velocity limit

$$
T_{\omega}^{\mu \nu} \approx T_{\Omega(1)}^{\mu \nu}+T_{\Omega(0)}^{\mu \nu}
$$

For convenience, we may call $T_{\Omega}^{\mu \nu} \triangleq T_{\Omega(1)}^{\mu \nu}+T_{\Omega(0)}^{\mu \nu}$ the contravariant energy-momentum tensor of vacuum. From Eqs. (84), we see that the tensor $T_{\omega}^{\mu \nu}$ is an estimation of $T_{\Omega}^{\mu \nu}$ when the velocity $u$ of the $\Omega(0)$ substratum is small comparing to $c$. Thus, we may call $T_{\omega}^{\mu \nu}$ the contravariant energy-momentum tensor of vacuum in the low velocity limit. We can verify that the field equations Eqs. (77) is invariant under the following gauge transformation $(23]$, p. $45 ; 24])$

$$
\psi^{\mu \nu} \rightarrow \psi^{\mu \nu}+\partial^{\mu} \Lambda^{\nu}+\partial^{\nu} \Lambda^{\mu}
$$

where $\Lambda^{\mu}$ is an arbitrary vector field.

We introduce the following definition

$$
\phi^{\mu \nu}=\psi^{\mu \nu}-\frac{1}{2} \eta^{\mu \nu} \psi
$$

Using Eqs. (86), the field equations (77) can be written as

$$
\begin{aligned}
\partial_{\lambda} \partial^{\lambda} \phi^{\mu \nu} & -\partial_{\lambda} \partial^{\mu} \phi^{\nu \lambda}-\partial_{\lambda} \partial^{\nu} \phi^{\mu \lambda} \\
& +\eta^{\mu \nu} \partial_{\sigma} \partial_{\lambda} \phi^{\sigma \lambda}=-f_{0}\left(T^{\mu \nu}-T_{\omega}^{\mu \nu}\right)
\end{aligned}
$$

We introduce the following Hilbert gauge condition [24]

$$
\partial_{\mu}\left(\psi^{\mu \nu}-\frac{1}{2} \eta^{\mu \nu} \psi\right)=0 .
$$

Using Eqs. (86), the Hilbert gauge condition Eqs. (88) simplifies to

$$
\partial_{\mu} \phi^{\mu \nu}=0
$$

Applying Eqs. (89) in Eqs. (87), we obtain the following proposition [24].

Proposition 2 If we impose the Hilbert gauge condition Eqs. (88) on the fields, then, the field equations Eqs. (77) simplifies to

$$
\partial_{\lambda} \partial^{\lambda}\left(\psi^{\mu \nu}-\frac{1}{2} \eta^{\mu \nu} \psi\right)=-f_{0}\left(T^{\mu \nu}-T_{\omega}^{\mu \nu}\right) .
$$

If the tensorial potential $\psi^{\mu \nu}$ does not satisfy the Hilbert gauge condition Eqs. (88), then we can always construct a new tensorial potential $\bar{\psi}^{\mu \nu}$ by the following gauge transformation [24]

$$
\bar{\psi}^{\mu \nu}=\psi^{\mu \nu}+\partial^{\mu} \Lambda^{\nu}+\partial^{\nu} \Lambda^{\mu},
$$

such that the new tensorial potential $\bar{\psi}^{\mu \nu}$ does satisfy the Hilbert gauge condition Eqs. (88). 
Using Eqs. (86), the field equations Eqs. (90) can be written as

$$
\partial_{\lambda} \partial^{\lambda} \phi^{\mu \nu}=-f_{0}\left(T^{\mu \nu}-T_{\omega}^{\mu \nu}\right) .
$$

The field equation Eqs. (92) can also be written as

$$
\eta^{\alpha \beta} \frac{\partial^{2} \phi^{\mu \nu}}{\partial x^{\alpha} \partial x^{\beta}}=-f_{0}\left(T^{\mu \nu}-T_{\omega}^{\mu \nu}\right) .
$$

We noticed that the tensorial field equations Eqs. (93) is similar to the wave equations of electromagnetic fields.

\section{CONSTRUCTION OF A TENSORIAL POTENTIAL IN INERTIAL COORDINATE SYSTEMS}

The existence of the $\Omega(1)$ substratum allows us to introduce the following definition of inertial coordinate systems.

Definition 1 If a coordinates system $S$ is static or moving with a constant velocity relative to the reference frame $S_{\Omega(1)}$, then, we call such a coordinates system as an inertial coordinate system.

The field equations Eqs. (87) and Eqs. (90) are valid in the reference frame $S_{\Omega(1)}$. We will explore the possibility of constructing a tensorial potential in an arbitrary inertial system $S^{\prime}$. In an inertial coordinate system $S$, an arbitrary event is characterized by the four space-time coordinates $(t, x, y, z)$. In an inertial system $S^{\prime}$, this event is characterized by four other coordinates $\left(t^{\prime}, x^{\prime}, y^{\prime}, z^{\prime}\right)$. We assume that the origins of the Cartesian coordinates in the two inertial systems $S$ and $S^{\prime}$ coincide at the time $t=t^{\prime}=0$. Then, the connections between these space-time coordinates are given by a homogeneous linear transformation keeping the quantity $s^{2}=c^{2} t^{2}-x^{2}-y^{2}-z^{2}$ invariant, i.e., ([26], p. 92)

$$
s^{2}=c^{2} t^{2}-x^{2}-y^{2}-z^{2}=c^{2} t^{\prime 2}-x^{\prime 2}-y^{\prime 2}-z^{\prime 2}=s^{\prime 2} .
$$

We introduce the following two coordinate systems

$$
\begin{aligned}
& x^{0}=c t, \quad x^{1}=x, \quad x^{2}=y, \quad x^{3}=z, \\
& x^{\prime 0}=c t^{\prime}, \quad x^{\prime 1}=x^{\prime}, \quad x^{\prime 2}=y^{\prime}, \quad x^{\prime 3}=z^{\prime} .
\end{aligned}
$$

The homogeneous linear transformation keeping the quantity $s^{2}$ invariant, which is usually called the Lorentz transformation, can be written as (33], p. 57; [26], p. 92)

$$
x^{\prime \mu}=\alpha^{\mu}{ }_{\nu} x^{\nu},
$$

where $\alpha^{\mu}{ }_{\nu}$ are coefficients depend only on the angles between the spatial axes in the two inertial systems $S$ and $S^{\prime}$ and on the relative velocity of $S$ and $S^{\prime}$.

Applying the standard methods in theory of special relativity [26], we have the following results.
Proposition 3 Suppose that the field equations Eqs. (92) is valid in the the reference frame $S_{\Omega(1)}$. Then, in an arbitrary inertial system $S^{\prime}$, there exists a symmetric tensor $\phi_{\mu \nu}^{\prime}$ satisfies the following wave equation

$$
\partial_{\lambda}^{\prime} \partial^{\prime \lambda} \phi^{\prime \mu \nu}=-f_{0}\left(T^{\prime \mu \nu}-T_{\omega}^{\prime \mu \nu}\right),
$$

where $T^{\prime \mu \nu}$ and $T_{\omega}^{\prime \mu \nu}$ are corresponding tensors of $T^{\mu \nu}$ and $T_{\omega}^{\mu \nu}$ in the arbitrary inertial coordinate system $S^{\prime}$ respectively.

Proposition 4 Suppose that the field equations Eqs. $87)$ is valid in the reference frame $S_{\Omega(1)}$. Then, in an arbitrary inertial system $S^{\prime}$, there exists a symmetric tensor $\phi_{\mu \nu}^{\prime}$ satisfies the following field equation

$$
\begin{aligned}
\partial_{\lambda}^{\prime} \partial^{\prime \lambda} \phi^{\prime \mu \nu} & -\partial_{\lambda}^{\prime} \partial^{\prime \mu} \phi^{\prime \nu \lambda}-\partial_{\lambda}^{\prime} \partial^{\prime \nu} \phi^{\prime \mu \lambda} \\
& +\eta^{\mu \nu} \partial_{\sigma}^{\prime} \partial_{\lambda}^{\prime} \phi^{\sigma \sigma \lambda}=-f_{0}\left(T^{\prime \mu \nu}-T_{\omega}^{\prime \mu \nu}\right) .
\end{aligned}
$$

\section{THE EQUATIONS OF MOTION OF A POINT PARTICLE IN A GRAVITATIONAL FIELD AND INTRODUCTION OF AN EFFECTIVE RIEMANNIAN SPACETIME}

In this section, we study the equations of motion of a free point particle in a gravitational field. The Lagrangian of a free point particle can be written as ([23], p. $57 ; 24]$ )

$$
L_{0}=\frac{1}{2} m \frac{d x^{\mu}}{d \tau} \frac{d x_{\mu}}{d \tau}=\frac{1}{2} m u^{\mu} u_{\mu}=\frac{1}{2} m \eta_{\mu \nu} u^{\mu} u^{\nu},
$$

where $m$ is the rest mass of the point particle, $d \tau \equiv$ $\frac{1}{c} \sqrt{d x^{\mu} d x_{\mu}}$ is the infinitesimal proper time interval, $u^{\mu} \equiv$ $d x^{\mu} / d \tau$.

Suppose that $T_{\Omega(1)}^{\mu \nu} \approx 0$. Ignoring those higher terms $O\left[\left(f_{0} \psi_{\mu \nu}\right)^{2}\right]$ in Eq. (51), the interaction term of the Lagrangian of a system of the $\Omega(0)$ substratum, the $\Omega(1)$ substratum and the point particle can be written in the following form (23], p. 57;24])

$$
L_{\text {int }}=f_{0} \psi_{\mu \nu} m u^{\mu} u^{\nu} .
$$

Using Eq. (100) and Eq. (99), the total Lagrangian $L_{\text {tot }}$ of a system of the $\Omega(0)$ substratum, the $\Omega(1)$ substratum and the point particle can be written as ([23], p. 57)

$$
L_{\mathrm{tot}}=L_{0}+L_{\mathrm{int}}=\frac{1}{2} m u^{\mu} u_{\mu}+f_{0} \psi_{\mu \nu} m u^{\mu} u^{\nu} .
$$

The Euler-Lagrange equations for the total Lagrangian $L_{\text {tot }}$ can be written as ([33],p. 111)

$$
\frac{d}{d \tau}\left[\left(\eta_{\mu \nu}+2 f_{0} \psi_{\mu \nu}\right) \frac{d x^{\nu}}{d \tau}\right]-f_{0} \frac{\partial \psi_{\alpha \beta}}{\partial x^{\mu}} \frac{d x^{\alpha}}{d \tau} \frac{d x^{\beta}}{d \tau}=0 .
$$

We notice that the equations of motion (102) of a point particle in gravitational field are similar to the equations of a geodesic line (104) in a Riemannian spacetime. Thus, 
it is natural for us to introduce the following definition of a metric tensor $g_{\mu \nu}$ of a Riemannian spacetime ([23], p. 57)

$$
g_{\mu \nu}=\eta_{\mu \nu}+2 f_{0} \psi_{\mu \nu}
$$

Then, the equations of motion Eqs. (102) can be written as $(23]$, p. 58)

$$
\frac{d}{d \tau_{g}}\left(g_{\mu \nu} \frac{d x^{\nu}}{d \tau_{g}}\right)=\frac{1}{2} \frac{\partial g_{\alpha \beta}}{\partial x^{\mu}} \frac{d x^{\alpha}}{d \tau_{g}} \frac{d x^{\beta}}{d \tau_{g}},
$$

where $\tau_{g}$ is the proper time interval in the Riemannian spacetime with a metric tensor $g_{\mu \nu}$.

Eqs. (104) represent a geodesic line in a Riemannian spacetime with a metric tensor $g_{\mu \nu}$, which can also be written as (34], p. 51)

$$
\frac{d^{2} x^{\mu}}{d \tau_{g}^{2}}+\Gamma_{\nu \sigma}^{\mu} \frac{d x^{\nu}}{d \tau_{g}} \frac{d x^{\sigma}}{d \tau_{g}}=0
$$

where

$$
\Gamma_{\alpha \beta}^{\nu} \triangleq \frac{1}{2} g^{\mu \nu}\left(\frac{\partial g_{\mu \alpha}}{\partial x^{\beta}}+\frac{\partial g_{\mu \beta}}{\partial x^{\alpha}}-\frac{\partial g_{\alpha \beta}}{\partial x^{\mu}}\right)
$$

are the Christoffel symbols.

Thus, we find that the equations of motion (102) of a point particle in gravitational field are approximately a geodesic line described in Eqs. (105) in a Riemannian spacetime with a metric tensor $g_{\mu \nu}$.

According to Assumption [1] the particles that constitute the $\Omega(1)$ substratum are sinks in the $\Omega(0)$ substratum. Thus, the movements of the $\Omega(1)$ substratum in gravitational field will be different from the Maxwell's equations. We notice that the equations of motion of a point particle in gravitational field (104) are generalizations of the equations of motion of a point particle in vacuum free of gravitational field. The law of propagation of an electromagnetic wave front in vacuum free of gravitational field is Eqs. (10). Thus, the law of propagation of an electromagnetic wave front in gravitational field may be a kind of generalization of Eq. (10). Therefore, we introduce the following assumption.

Assumption 6 To first order of $f_{0} \psi_{\mu \nu}$, the law of propagation of an electromagnetic wave front $\omega\left(x^{0}, x^{1}, x^{2}, x^{3}\right)$ in gravitational field is

$$
g_{\mu \nu} \frac{\partial \omega}{\partial x^{\mu}} \frac{\partial \omega}{\partial x^{\nu}}=0
$$

where $\omega\left(x^{0}, x^{1}, x^{2}, x^{3}\right)$ is the electromagnetic wave front, $g_{\alpha \beta}$ is the metric tensor defined in Eqs. (103).

The measurements of spacetime intervals are carried out using light rays and point particles, which are only subject to inertial force and gravitation. Thus, according to Eqs. (104) and Eq. (107), the physically observable metric of spacetime, to first order of $f_{0} \psi_{\mu \nu}$, is $g_{\mu \nu}$. Thus, the initial flat background spacetime with metric $\eta_{\mu \nu}$ is no longer physically observable [24].

If we can further derive the Einstein's equations (1) using the definition (103) of a metric tensor $g_{\mu \nu}$ of a Riemannian spacetime, then, we may provide a geometrical interpretation of Einstein's theory of gravitation based on the theory of vacuum mechanics [15, 21, 22]. This is the task of the next section.

\section{DERIVATION OF GENERALIZED EINSTEIN EQUATIONS IN INERTIAL COORDINATE SYSTEMS}

Definition 2 The Einstein tensor $G_{\mu \nu}$ is defined by

$$
G_{\mu \nu} \triangleq R_{\mu \nu}-\frac{1}{2} g_{\mu \nu} R
$$

where $g_{\mu \nu}$ is a metric tensor of a Riemannian spacetime, $R_{\mu \nu}$ is the Ricci tensor, $R \triangleq g^{\mu \nu} R_{\mu \nu}, g^{\mu \nu}$ is the corresponding contravariant tensor of $g_{\mu \nu}$ such that $g_{\mu \lambda} g^{\lambda \nu}=\delta_{\mu}^{\nu}=g_{\mu}^{\nu}$ (34], p. 40).

According to the geometrical interpretation of some theories of gravitation in flat spacetime [24], the physically observable metric $g_{\mu \nu}$ of spacetime in Eqs. (103) can be written as

$$
g^{\mu \nu}=\eta^{\mu \nu}-2 f_{0} \psi^{\mu \nu}+O\left[\left(f_{0} \psi^{\mu \nu}\right)^{2}\right] .
$$

Following the clue showed in Eqs. (109) and noticing the methods of S. N. Gupta [35] and W. Thirring [24], we introduce the following definition of a metric tensor of a Riemannian spacetime.

\section{Definition 3}

$$
\tilde{g}^{\mu \nu} \triangleq \sqrt{-g_{0}} g^{\mu \nu} \triangleq \eta^{\mu \nu}-2 f_{0} \phi^{\mu \nu},
$$

where $g_{0}=$ Det $g_{\mu \nu}$.

We have the following expansion of the contravariant metric tensor $g^{\mu \nu}$ 35]

$$
\begin{aligned}
g^{\mu \nu}= & \eta^{\mu \nu}-2 f_{0} \phi^{\mu \nu}+f_{0} \eta^{\mu \nu} \eta_{\alpha \beta} \phi^{\alpha \beta} \\
& -2 f_{0}^{2} \eta_{\alpha \beta} \phi^{\alpha \beta} \phi^{\mu \nu}+f_{0}^{2} \eta^{\mu \nu} \eta_{\alpha \sigma} \eta_{\beta \lambda} \phi^{\alpha \beta} \phi^{\lambda \sigma} \\
& +\frac{1}{2} f_{0}^{2} \eta^{\mu \nu} \eta_{\alpha \beta} \eta_{\lambda \sigma} \phi^{\alpha \beta} \phi^{\lambda \sigma}+O\left[\left(f_{0} \phi^{\mu \nu}\right)^{3}\right] .(11
\end{aligned}
$$

Definition 4 If $\phi^{\mu \nu}$ and their first and higher derivatives satisfy the following conditions

$$
\begin{aligned}
& \left|2 f_{0} \phi^{\mu \nu}\right| \ll 1 \\
& \left|\frac{\partial^{n}\left(2 f_{0} \phi^{\mu \nu}\right)}{\partial\left(x^{\alpha}\right)^{n}}\right| \ll 1, n=1,2,3, \cdots
\end{aligned}
$$

then we call this filed $\phi^{\mu \nu}$ weak.

For weak fields, $\psi \approx \phi \approx 0$. Thus, $\phi^{\mu \nu}=\psi^{\mu \nu}-1 / 2$. $\eta^{\mu \nu} \psi \approx \psi^{\mu \nu}$. From Eqs. (111), we see that the definition (110) is compatible with Eqs. (109). 
Theorem 3 Suppose that Assumption 5 is valid. Then, in an arbitrary inertial coordinate system $S_{i}$, we have the following field equations

$$
\begin{aligned}
G^{\mu \nu} & -\frac{1}{2 g_{0}}\left(\sqrt{-g_{0}} g^{\alpha \beta}-\eta^{\alpha \beta}\right) \frac{\partial^{2}\left(\sqrt{-g_{0}} g^{\mu \nu}\right)}{\partial x^{\alpha} \partial x^{\beta}} \\
& -\frac{\sqrt{-g_{0}}}{2 g_{0}}\left(\partial_{\lambda} \partial^{\mu} g^{\nu \lambda}+\partial_{\lambda} \partial^{\nu} g^{\mu \lambda}-\eta^{\mu \nu} \partial_{\sigma} \partial_{\lambda} g^{\sigma \lambda}\right) \\
& -\Pi^{\mu, \alpha \beta} \Pi_{\alpha \beta}^{\nu}+\frac{1}{2} y^{\mu} y^{\nu}-\frac{1}{2} g^{\mu \nu}(L+B) \\
& +B^{\mu \nu}=\frac{f_{0}^{2}}{g_{0}}\left(T^{\mu \nu}-T_{\omega}^{\mu \nu}\right)
\end{aligned}
$$

where $T^{\mu \nu}$ is the contravariant total energy-momentum tensor of the system of the matter, the $\Omega(1)$ substratum and the $\Omega(0)$ substratum in the inertial coordinate system $S_{i}, T_{\omega}^{\mu \nu}$ is the contravariant energy-momentum tensor of vacuum in the low velocity limit in $S_{i}$,

$$
\begin{gathered}
\Pi^{\mu, \alpha \beta} \frac{1}{2 g_{0}}\left(\tilde{g}^{\alpha \lambda} \frac{\partial \tilde{g}^{\mu \beta}}{\partial x^{\lambda}}+\tilde{g}^{\beta \lambda} \frac{\partial \tilde{g}^{\mu \alpha}}{\partial x^{\lambda}}-\tilde{g}^{\mu \lambda} \frac{\partial \tilde{g}^{\alpha \beta}}{\partial x^{\lambda}}\right) \\
\Pi_{\alpha \beta}^{\nu} \triangleq g_{\alpha \lambda} g_{\beta \sigma} \Pi^{\nu, \lambda \sigma} \\
\Gamma^{\mu \nu} \triangleq \frac{1}{2}\left(g^{\mu \alpha} \frac{\partial \Gamma^{\nu}}{\partial x^{\alpha}}+g^{\nu \alpha} \frac{\partial \Gamma^{\mu}}{\partial x^{\alpha}}-\frac{\partial g^{\mu \nu}}{\partial x^{\alpha}} \Gamma^{\alpha}\right) \\
y_{\beta} \triangleq \frac{\partial\left(\lg \sqrt{-g_{0}}\right)}{\partial x^{\beta}}, y^{\alpha} \triangleq g^{\alpha \beta} y_{\beta} \\
L \triangleq-\frac{1}{2} \Gamma_{\alpha \beta}^{\nu} \frac{\partial g^{\alpha \beta}}{\partial x^{\nu}}-\Gamma^{\alpha} \frac{\partial\left(\lg \sqrt{-g_{0}}\right)}{\partial x^{\alpha}} \\
B^{\mu \nu} \triangleq \Gamma^{\mu \nu}+\frac{1}{2}\left(y^{\mu} \Gamma^{\nu}+y^{\nu} \Gamma^{\mu}\right), B \triangleq g_{\mu \nu} B^{\mu \nu}
\end{gathered}
$$

Proof of Theorem [3. According to a theorem of V. Fock ([27], p. 429), the contravariant Einstein tensor $G^{\mu \nu}$ can be written as

$$
\begin{aligned}
G^{\mu \nu}= & \frac{1}{2 g_{0}} \tilde{g}^{\alpha \beta} \frac{\partial^{2} \tilde{g}^{\mu \nu}}{\partial x_{\alpha} \partial x_{\beta}}+\Pi^{\mu, \alpha \beta} \Pi_{\alpha \beta}^{\nu}-\frac{1}{2} y^{\mu} y^{\nu} \\
& +\frac{1}{2} g^{\mu \nu}(L+B)-B^{\mu \nu}
\end{aligned}
$$

Applying Eqs. (110), Eqs. (122) can be written as

$$
\begin{aligned}
G^{\mu \nu}= & \frac{1}{2 g_{0}}\left(\sqrt{-g_{0}} g^{\alpha \beta}-\eta^{\alpha \beta}\right) \frac{\partial^{2}\left(-2 f_{0} \phi^{\mu \nu}\right)}{\partial x^{\alpha} \partial x^{\beta}} \\
& -\frac{f_{0}}{g_{0}} \eta^{\alpha \beta} \frac{\partial^{2} \phi^{\mu \nu}}{\partial x^{\alpha} \partial x^{\beta}}+\Pi^{\mu, \alpha \beta} \Pi_{\alpha \beta}^{\nu} \\
& -\frac{1}{2} y^{\mu} y^{\nu}+\frac{1}{2} g^{\mu \nu}(L+B)-B^{\mu \nu} .
\end{aligned}
$$

Noticing Eqs. (110), the field equations Eqs. (98) can be written as

$$
\begin{aligned}
\eta^{\alpha \beta} \frac{\partial^{2} \phi^{\mu \nu}}{\partial x^{\alpha} \partial x^{\beta}}= & -\frac{\sqrt{-g_{0}}}{2 f_{0}}\left(\partial_{\lambda} \partial^{\mu} g^{\nu \lambda}+\partial_{\lambda} \partial^{\nu} g^{\mu \lambda}\right. \\
& \left.-\eta^{\mu \nu} \partial_{\sigma} \partial_{\lambda} g^{\sigma \lambda}\right)-f_{0}\left(T^{\mu \nu}-T_{\omega}^{\mu \nu}(1.24)\right.
\end{aligned}
$$

Using Eqs. (110) and Eqs. (124), Eqs. (123) can be written as

$$
\begin{aligned}
G^{\mu \nu}= & \frac{1}{2 g_{0}}\left(\sqrt{-g_{0}} g^{\alpha \beta}-\eta^{\alpha \beta}\right) \frac{\partial^{2}\left(\sqrt{-g_{0}} g^{\mu \nu}\right)}{\partial x^{\alpha} \partial x^{\beta}} \\
& +\frac{\sqrt{-g_{0}}}{2 g_{0}}\left(\partial_{\lambda} \partial^{\mu} g^{\nu \lambda}+\partial_{\lambda} \partial^{\nu} g^{\mu \lambda}-\eta^{\mu \nu} \partial_{\sigma} \partial_{\lambda} g^{\sigma \lambda}\right) \\
& +\frac{f_{0}^{2}}{g_{0}}\left(T^{\mu \nu}-T_{\omega}^{\mu \nu}\right)+\Pi^{\mu, \alpha \beta} \Pi_{\alpha \beta}^{\nu}-\frac{1}{2} y^{\mu} y^{\nu} \\
& +\frac{1}{2} g^{\mu \nu}(L+B)-B^{\mu \nu} .
\end{aligned}
$$

Eqs. (125) can be written as Eqs. (114).

Eqs. (114) have the same form in all inertial coordinate systems. Eqs. (114) is one of the main results in this manuscript. We need to further study the relationship between Eqs. (114) and the Einstein field equations Eqs. (11).

Theorem 4 If we impose the Hilbert gauge Eqs. (88) on the fields, then in an arbitrary inertial coordinate system $S_{i}$ we have the following field equations

$$
\begin{aligned}
G^{\mu \nu} & -\frac{1}{2 g_{0}}\left(\sqrt{-g_{0}} g^{\alpha \beta}-\eta^{\alpha \beta}\right) \frac{\partial^{2}\left(\sqrt{-g_{0}} g^{\mu \nu}\right)}{\partial x^{\alpha} \partial x^{\beta}} \\
& -\Pi^{\mu, \alpha \beta} \Pi_{\alpha \beta}^{\nu}+\frac{1}{2} y^{\mu} y^{\nu}-\frac{1}{2} g^{\mu \nu}(L+B) \\
& +B^{\mu \nu}=\frac{f_{0}^{2}}{g_{0}}\left(T^{\mu \nu}-T_{\omega}^{\mu \nu}\right) .
\end{aligned}
$$

Proof of Theorem 4. Using Eqs. (110) and Eqs. (97), Eqs. (123) can be written as

$$
\begin{aligned}
G^{\mu \nu}= & \frac{1}{2 g_{0}}\left(\sqrt{-g_{0}} g^{\alpha \beta}-\eta^{\alpha \beta}\right) \frac{\partial^{2}\left(\sqrt{-g_{0}} g^{\mu \nu}\right)}{\partial x^{\alpha} \partial x^{\beta}} \\
& +\frac{f_{0}^{2}}{g_{0}}\left(T^{\mu \nu}-T_{\omega}^{\mu \nu}\right)+\Pi^{\mu, \alpha \beta} \Pi_{\alpha \beta}^{\nu}-\frac{1}{2} y^{\mu} y^{\nu} \\
& +\frac{1}{2} g^{\mu \nu}(L+B)-B^{\mu \nu} .
\end{aligned}
$$

Eqs. (127) can be written as Eqs. (126).

Definition 5 If each of the coordinates $x^{\alpha}$ satisfies the following generalized wave equations

$$
\frac{1}{\sqrt{-g_{0}}} \frac{\partial}{\partial x^{\mu}}\left(\sqrt{-g_{0}} g^{\mu \nu} \frac{\partial x^{\alpha}}{\partial x^{\nu}}\right)=0,
$$

then, we call such a coordinates system harmonic. 
In a harmonic coordinates system, we have ([27], p. 254)

$$
\Gamma^{\nu}=\Gamma^{\mu \nu}=B^{\mu \nu}=B=0 .
$$

Putting Eqs. (129) into Eqs. (126), we have the following corollary.

Corollary 1 If we apply the Hilbert gauge Eqs. (88) and the coordinates system is harmonic, then the field equations Eqs. (126) can be written as

$$
\begin{aligned}
G^{\mu \nu} & -\frac{1}{2 g_{0}}\left(\sqrt{-g_{0}} g^{\alpha \beta}-\eta^{\alpha \beta}\right) \frac{\partial^{2}\left(\sqrt{-g_{0}} g^{\mu \nu}\right)}{\partial x^{\alpha} \partial x^{\beta}} \\
& -\Pi^{\mu, \alpha \beta} \Pi_{\alpha \beta}^{\nu}+\frac{1}{2} y^{\mu} y^{\nu}-\frac{1}{2} g^{\mu \nu} L \\
& =\frac{f_{0}^{2}}{g_{0}}\left(T^{\mu \nu}-T_{\omega}^{\mu \nu}\right) .
\end{aligned}
$$

We can verify that each of the Galilean coordinates is harmonic. Any constant and any linear function of harmonic coordinates satisfy Eqs. (128). Thus, from Eqs. (96) we see that an inertial coordinate system is harmonic and Eqs. (130) are valid for every inertial system. In order to study the case of weak fields in inertial systems, we introduce the following assumption.

Assumption 7 Suppose that the dimensionless parameter $\varpi=m_{0} c / 2 \rho_{0} q_{0}$ satisfies the following condition

$$
\varpi=\frac{m_{0} c}{2 \rho_{0} q_{0}} \leq 1 .
$$

Using the 00 component of Eqs. (113) for the case $n=$ 1 and noticing Eqs. (86), Eqs. (28), Eqs. (50) and Eqs. (8), we have

$$
\left|\frac{\partial\left(2 f_{0} \phi^{00}\right)}{\partial\left(x^{\alpha}\right)}\right|=\left|\frac{2 \rho_{0} q_{0}}{m_{0} c^{2}} \frac{\partial \varphi}{\partial x^{\alpha}}\right| \ll 1 .
$$

Noticing Eq. (26) and using Eq. (132) and Eq. (131), we have $|u| \approx|\nabla \varphi| \ll m_{0} c^{2} /\left(2 \rho_{0} q_{0}\right) \leq c$. Therefore, according to Assumption 5. Eqs. (74) and Eqs. (76) are valid for weak fields.

Corollary 2 Suppose that (1) the Hilbert gauge Eqs. (88) is applied on the fields; (2) the filed is weak; (3) Assumption 5 is valid. Then in an arbitrary inertial coordinate system the field equations Eqs. (130) reduce to

$$
R_{\mu \nu}-\frac{1}{2} g_{\mu \nu} R=\frac{f_{0}^{2}}{g_{0}} T_{\mu \nu}^{m}
$$

Proof of Corollary 2, According to Definition $4 f_{0} \phi^{\mu \nu}$ and their first and higher derivatives are small quantities of order $\varepsilon$, where $|\varepsilon| \ll 1$ is a small quantity. Thus, using Eqs. (110) and Eqs. (111), we have the following estimation of the order of magnitude of the following quantities

$$
\sqrt{-g_{0}} g^{\mu \nu}-\eta^{\mu \nu} \sim \varepsilon, \quad \frac{\partial g_{\mu \nu}}{\partial x^{\alpha}} \sim \frac{\partial g^{\mu \nu}}{\partial x^{\alpha}} \sim \varepsilon .
$$

From Eqs. (110), we have the following estimation of the order of magnitude of the quantity

$$
\frac{\partial^{2}\left(\sqrt{-g_{0}} g^{\alpha \beta}\right)}{\partial x^{\alpha} \partial x^{\beta}}=\frac{\partial^{2}\left(-2 f_{0} \phi^{\mu \nu}\right)}{\partial x^{\alpha} \partial x^{\beta}} \sim \varepsilon .
$$

Thus, using Eqs. (134) and Eqs. (135), we have the following estimation of the order of magnitude of the quantity

$$
\left(\sqrt{-g_{0}} g^{\alpha \beta}-\eta^{\alpha \beta}\right) \frac{\partial^{2}\left(\sqrt{-g_{0}} g^{\alpha \beta}\right)}{\partial x^{\alpha} \partial x^{\beta}} \sim \varepsilon^{2} .
$$

From Eqs. (115) and Eqs. (116), we have the following estimation of the order of magnitude of the following quantities

$$
\Pi^{\mu, \alpha \beta} \sim \Pi_{\alpha \beta}^{\nu} \sim \varepsilon .
$$

Using Eqs. (119), we have the following relationship (27], p. 143)

$$
y_{\beta}=\Gamma_{\beta \nu}^{\nu} .
$$

We also have ([27], p. 143)

$$
\Gamma_{\beta \nu}^{\nu}=\frac{1}{2} g^{\mu \nu} \frac{\partial g_{\mu \nu}}{\partial x^{\beta}} .
$$

From Eqs. (138), Eqs. (139) and Eqs. (134), we have the following estimation of the order of magnitude

$$
y_{\beta} \sim \varepsilon .
$$

Using Eqs. (119) and Eqs. (140), we have the following estimation of the order of magnitude

$$
y^{\alpha} \sim \varepsilon .
$$

From Eq. (120) and Eqs. (129), we have

$$
L=-\frac{1}{2} \Gamma_{\alpha \beta}^{\nu} \frac{\partial g^{\alpha \beta}}{\partial x^{\nu}} .
$$

Using Eq. (142), Eqs. (106) and Eqs. (134), we have the following estimation of the order of magnitude

$$
L \sim \varepsilon^{2} .
$$

From Eqs. (136), Eqs. (137), Eqs. (141) and Eq. (143), we see that the second to the fifth term on the right side of Eqs. (130) are all small quantities of order $\varepsilon^{2}$. Ignoring all these small quantities of order $\varepsilon^{2}$ in Eqs. (130) and using Eqs. (76), we obtain

$$
G^{\mu \nu} \approx \frac{f_{0}^{2}}{g_{0}} T_{m}^{\mu \nu}
$$

Applying the rules of lowering or raising the indexes of tensors, i.e., $G^{\mu \nu}=g^{\mu \lambda} g^{\nu \sigma} G_{\lambda \sigma}, T_{m}^{\mu \nu}=g^{\mu \lambda} g^{\nu \sigma} T_{\lambda \sigma}^{m}$, Eqs. (144) can be written as

$$
G_{\lambda \sigma} \approx \frac{f_{0}^{2}}{g_{0}} T_{\lambda \sigma}^{m}
$$

Putting Eqs. (108) into Eqs. (145), we obtain Eqs. (133). 
Corollary 3 Suppose that the following conditions are valid: (1) the Hilbert gauge Eqs. (88) is applied on the fields; (2) the filed is weak; (3) $g_{0} \approx-1$; (4) Assumption 5 is valid. Then in an arbitrary inertial coordinate system the field equation Eqs. (139) reduce to

$$
R_{\mu \nu}-\frac{1}{2} g_{\mu \nu} R=-f_{0}^{2} T_{\mu \nu}^{m} .
$$

If we introduce the following notation

$$
\kappa=f_{0}^{2},
$$

then, Eqs. 146) coincide with Einstein's equations Eqs. (11). Thus, we see that the field equations Eqs. (114) are generalizations of the Einstein's equations Eqs. (11) in inertial coordinate systems.

\section{CONCLUSION}

We extend our previous theory of gravitation based on a sink flow model of particles by methods of special relativistic fluid mechanics. In inertial coordinate systems, we construct a tensorial potential of the $\Omega(0)$ substratum. Based on some assumptions, we show that this tensorial potential satisfies the wave equations. Inspired by the equations of motion of a test particle, a definition of a metric tensor of a Riemannian spacetime is introduced. Generalized Einstein's equations in inertial coordinate systems are derived based on some assumptions. These equations reduce to the Einstein's equations in case of weak field in harmonic coordinate systems. In our theory, gravity is transmitted by the $\Omega(0)$ substratum. The theory of general relativity can not provide a physical definition of the metric tensor of the Riemannian spacetime. In our theory, the background spacetime is the Minkowshi spacetime. However, the flat background spacetime is no longer physically observable. According to the equations of motion of a point particle in gravitational field, to first order, the physically observable spacetime is a Riemannian spacetime. The metric tensor of this Riemannian spacetime is defined based on the tensorial potential of gravitational fields.

\section{Acknowledgments}

This work was partly supported by the Doctor Research Foundation of Henan Polytechnic University (Grant No. B2012-069). I would like to thank an anonymous reviewer for pointing out some mistakes and providing some suggestions.
[1] S. Weinberg, Gravitation and cosmology (John Wiley \& sons, New York, 1972).

[2] C. Misner, K. Thorne, and J. Wheeler, Gravitation (Freeman, New York, 1973).

[3] A. Hees and et al., Phys. Rev. Lett. 118, 211101 (2017).

[4] J. Maddox, What remains to be discovered (The Free Press, New York, 1998).

[5] D. Marsh, Phys. Rev. Lett. 118, 011302 (2017).

[6] A. Cho, Science 355, 560 (2017).

[7] P. Dirac, Directions in Physics (John Wiley \& sons, New York, 1978).

[8] J. Kim, P. Sikivie, and S. Weinberg, Nature 541, 464 (2017).

[9] M. Farhoudi and M. Yousefian, Int. J. Theor. Phys. 55, 2436 (2016).

[10] D. R. Long, Nature 260, 417 (1976).

[11] A. Balcerzak, M. P. Dabrowski, and V. Salzano, Annalen der Physik 529, 1600409 (2017).

[12] N. Cornish, D. Blas, and G. Nardini, Phys. Rev. Lett. 119, 161102 (2017).

[13] D. Giovannini, J. Romero, V. Potocek, G. Ferenczi, F. Speirits, S. Barnett, D. Faccio, and M. Padgett, Science 347, 857 (2015).

[14] J. R. Sambles, Science 347, 828 (2015).

[15] X.-S. Wang, Progress in Physics 4, 25 (2008).

[16] E. Whittaker, A History of the Theories of Aether and Electricity, Revised and enlarged edtion, vol. 1 (Thomas Nelson and Sons Ltd., London, 1951).

[17] B. Riemann, Collected Papers, Translated from the 1892 edition by Roger Baker, Charles Christenson and Henry Orde (Kendrick Press, Heber, 2004).
[18] H. Poincare, The science and hypothesis, translated from the French edtion, in Chinese (Shangwu Press, Beijing, 1997).

[19] A. Einstein and L. Infeld, The evolution of physics (Cambridge University Press, London, 1938).

[20] I. G. Currie, Fundamental Mechanics of Fluids (Cambridge University Press, 2003), 3rd ed.

[21] X.-S. Wang, Progress in Physics 2, 111 (2008).

[22] X.-S. Wang, Physics Essays 27, 398 (2014).

[23] R. P. Feynman, Feynman lectures on gravitation, foreword by John Preskill and Kip S. Thorne (AddisonWesley Publishing Company, New York, 1995).

[24] W. Thirring, Annals of Physics 16, 96 (1961).

[25] S. Weinberg, Physical Review 138, B988 (1965).

[26] C. Moller, The theory of relativity (Clarendon Press, Oxford, 1955).

[27] V. Fock, The Theory of Space Time and Gravitation, 2nd Revised Edition, Translated from the Russian by N. Kemmer (Pergamon Press, London, 1964).

[28] W. Rindler, Introduction to special relativity (Clarendon Press, Oxford, 1982).

[29] Z.-D. Chen, Rational mechanics, in Chinese (Chongqing Press, Chongqing, 2000).

[30] L. D. Landau and E. M. Lifshitz, Fluid Mechanics, 2nd Edition, translated from the Russian by J. B. Sykes and W. H. Reid (Pergamon Press, Oxford, 1987).

[31] A. C. Eringen, Elastodynamics (Academic Press, New York, 1975).

[32] S. V. Babak and L. P. Grishchuk, Physical Review D 61, 024038 (1999).

[33] H. C. Ohanian and R. Ruffini, Gravitation and Space- 
time, 3rd edition (Cambridge University Press, New York, 2013).

[34] L. Liu, The theory of general relativity, in Chinese, 2nd edition (Advanced Education Press, Beijing, 2004). [35] S. N. Gupta, Proc. Phys. Soc. A65, 608 (1952). 